Functional Analysis and Its Applications, Vol. 40, No. 4, pp. 304-312, 2006

Translated from Funktsional'nyi Analiz i Ego Prilozheniya, Vol. 40, No. 4, pp. 72-82, 2006

Original Russian Text Copyright (c) by I. V. Ostrovskii and A. E. Üreyen

\title{
The Growth Irregularity of Slowly Growing Entire Functions
}

\section{V. Ostrovskii and A. E. Üreyen}

Received March 15, 2006

Dedicated to the centenary of Boris Yakovlevich Levin

ABSTRACT. We show that entire transcendental functions $f$ satisfying

$$
\log M(r, f)=o\left(\log ^{2} r\right), \quad r \rightarrow \infty \quad\left(M(r, f):=\max _{|z|=r}|f(z)|\right)
$$

necessarily have growth irregularity, which increases as the growth diminishes. In particular, if $1<p<2$, then the asymptotics

$$
\log M(r, f)=\log ^{p} r+o\left(\log ^{2-p} r\right), \quad r \rightarrow \infty,
$$

is impossible. It becomes possible if " $o$ " is replaced by " $O$."

KeY words: Clunie-Kövari theorem, Erdös-Kövari theorem, Hayman convexity theorem, maximum term, Levin's strong proximate order.

\section{Introduction and Statement of Results}

Let $\mathscr{A}$ be the set of all increasing functions $V$ defined for $r>0$, convex in $\log r$, and satisfying the condition

$$
\lim _{r \rightarrow \infty} \frac{V(r)}{\log r}=\infty
$$

Let $f$ be a transcendental entire function, and let

$$
M(r, f)=\max _{|z| \leqslant r}|f(z)| .
$$

The maximum modulus principle and the Hadamard three circles theorem imply that $\log M(r, f)$ $\in \mathscr{A}$. It is well known that $\mathscr{A}$ is wider than the set of all functions of the form $\log M(r, f)$. The following specific property of the latter can be mentioned: $\log M(r, f)$ must be piecewise analytic (e.g., see [11, p. 14] or [6, p. 11]). The problem of the asymptotic approximation of a function $V \in \mathscr{A}$ at $\infty$ by functions of form $\log M(r, f)$ can be viewed as the problem of existence of an entire function with prescribed growth. From this point of view, the problem had been studied by Edrei and Fuchs, Clunie, and Clunie and Kövari (see [4] and references therein). The most complete results are contained in [4].

The following theorem describes the best rate of asymptotic approximation to an arbitrary $V \in \mathscr{A}:$

Theorem A (Clunie-Kövari [4]). (i) For each $V \in \mathscr{A}$, there exists an entire function $f$ such that

$$
|\log M(r, f)-V(r)| \leqslant \frac{1}{2} \log r+O(1), \quad r \rightarrow \infty .
$$

(ii) The previous assertion ceases to be true if one replaces $1 / 2$ by any smaller constant.

In the theory of entire functions, the growth of an entire function is measured with the help of regularly growing functions $V$ of $\mathscr{A}$. The most important of these are

$$
V(r)=r^{\rho}, r^{\rho}(\log r)^{\rho_{1}}(\log \log r)^{\rho_{2}}, \ldots,
$$

and, more generally, $V(r)=r^{\rho(r)}$, where $\rho(r)$ is a proximate order (e.g., see [11, Sec. 6], [3, Sec. 4.22], and [9, Ch. 1, Sec. 12]). For regularly growing $V$, the rate of asymptotic approximation to $V$ by $\log M(r, f)$ can be viewed as a measure of possible growth regularity of an entire function. 
For the case in which $V$ does not increase too slowly, a solution to the problem is given by the following theorem:

Theorem B (Clunie-Kövari [4]). Under the additional assumption that the function $\psi(r):=$ $(d / d \log r) V(r)$ satisfies the condition

$$
\psi(c r)-\psi(r) \geqslant 1, \quad r \geqslant 1,
$$

for some $c>1$, there exists an entire function $f$ such that

$$
\log M(r, f)-V(r)=O(1), \quad r \rightarrow \infty .
$$

Condition (3) implies that

$$
\liminf _{r \rightarrow \infty} V(r)(\log r)^{-2}>0
$$

Therefore Theorem B does not apply to functions $V \in \mathscr{A}$ such that

$$
V(r)=o\left(\log ^{2} r\right), \quad r \rightarrow \infty .
$$

The present paper deals exactly with this case. The main result shows, in particular, that (4) is impossible here. Moreover, the slower the growth of a regularly growing $V$, the worse is the rate of its asymptotic approximation by functions of the form $\log M(r, f)$. In other words, if

$$
\log M(r, f)=o\left(\log ^{2} r\right), \quad r \rightarrow \infty,
$$

then the slower the growth of the transcendental entire function $f$, the more irregular it is. For example, our main theorem readily implies the impossibility of Eqs. (10)-(12) below.

Note that in the theory of entire functions there are many facts of opposite character: the slower the growth of $\log M(r, f)$, the more the asymptotic behavior of $f$ at $\infty$ resembles that of a polynomial. Let us mention, say, Wiman's theorem on functions of order less than $1 / 2$ and theorems on functions of order zero ([11, Secs. 9, 15, 16, 26]). Therefore, one might expect that as the growth of $\log M(r, f)$ diminishes, its regularity increases. The results of our paper show that this is not the case for entire functions satisfying (6).

Before stating our main theorem, we need to define a subset $\mathscr{A}_{\text {reg }}$ of $\mathscr{A}$ whose elements will be considered as regularly growing functions.

It is more convenient to change the scale by setting $\log r=x$. If $f$ is an entire transcendental function satisfying (6), then $\log M\left(e^{x}, f\right)$ has growth (as a function of $x$ ) not less than of order 1 and maximal type and not greater than of order 2 and minimal type. By Levin's theorem ([9, p. 39]), there exists a function $\lambda(x)$ (called a strong proximate order) of the form

$$
\lambda(x)=\lambda+\frac{\vartheta_{1}(\log x)-\vartheta_{2}(\log x)}{\log x},
$$

where $1 \leqslant \lambda \leqslant 2$ and $\vartheta_{j} \in C^{2}\left(\mathbb{R}_{+}\right)(j=1,2)$ is a concave function that satisfies the conditions

(a) $\lim _{x \rightarrow \infty} \vartheta_{j}(x)=\infty$

(b) $\lim _{x \rightarrow \infty} \vartheta_{j}(x) / x=0$, and

(c) $\lim _{x \rightarrow \infty} \vartheta_{j}^{\prime \prime}(x) / \vartheta_{j}^{\prime}(x)=0$,

such that

$$
\limsup _{x \rightarrow \infty} \frac{\log M\left(e^{x}, f\right)}{x^{\lambda(x)}}=1
$$

Note that Levin's construction shows that in the case of maximal (minimal) type of order $\lambda$ one can take $\vartheta_{2} \equiv 0\left(\vartheta_{1} \equiv 0\right)$, and we shall do this.

Definition. We denote by $\mathscr{A}_{\text {reg }}$ the set of all functions $V$ representable in the form

$$
V(r)=w(\log r),
$$

where $w$ is defined by

$$
w(x):=x^{\lambda} e^{\vartheta_{1}(\log x)-\vartheta_{2}(\log x)},
$$

$1 \leqslant \lambda \leqslant 2, \vartheta_{1}$ and $\vartheta_{2}$ have properties (a)-(c), and moreover, $\vartheta_{2} \equiv 0$ for $\lambda=1$ and $\vartheta_{1} \equiv 0$ for $\lambda=2$. 
Note that conditions (a)-(c) do not depend on the behavior of $\vartheta_{j}$ on any interval bounded from the right. Therefore, any pair of increasing concave functions $\vartheta_{1}, \vartheta_{2}$ defined only for sufficiently large $x$ and satisfying these conditions can be extended to $\mathbb{R}_{+}$in such a way that Eqs. (7) and (8) give a function $V \in \mathscr{A}_{\text {reg }}$. Hence it suffices to define a function $V \in \mathscr{A}_{\text {reg }}$ only for sufficiently large $r \in \mathbb{R}_{+}$.

The simplest examples of $V \in \mathscr{A}_{\text {reg }}$ are functions defined for sufficiently large $r$ in the following way:

$$
V(r)=(\log r)^{p_{1}}\left(\log _{2} r\right)^{p_{2}} \ldots\left(\log _{m} r\right)^{p_{m}},
$$

where $\log _{k}$ denotes the $k^{\text {th }}$ iteration of $\log$ and $p_{1}, \ldots, p_{m} \in \mathbb{R}$ are chosen in such a way that (1) and (5) are satisfied.

The main result of this paper is the following.

Theorem 1. (i) For any function $V \in \mathscr{A}_{\mathrm{reg}}$, let $w(x)=V\left(e^{x}\right)$. There does not exist an entire function $f$ such that

$$
\log M\left(e^{x}, f\right)-w(x)=o\left(\min \left(1 / w^{\prime \prime}(x), x\right)\right), \quad x \rightarrow \infty .
$$

(ii) The previous assertion ceases to be true if one replaces "o" by "O."

Since $\lim _{x \rightarrow \infty} w^{\prime \prime}(x)=0$ (see Lemma 2.1 below), assertion (i) (see Eq. (9)) implies that (4) is impossible for any $V \in \mathscr{A}_{\mathrm{reg}}$. The following theorem is an immediate corollary of Theorem 1 with

$$
\begin{gathered}
w(x)=x^{p}(\log x)^{\alpha}, \quad 1<p<2, \alpha \in \mathbb{R}, \\
w(x)=x(\log x)^{\alpha}, \quad \alpha>0, \quad w(x)=x^{2}(\log x)^{\alpha}, \quad \alpha<0 .
\end{gathered}
$$

Theorem 2. (i) No entire function $f$ can satisfy any of the following conditions $($ as $r \rightarrow \infty)$ :

$$
\begin{gathered}
\log M(r, f)-(\log r)^{p}\left(\log _{2} r\right)^{\alpha}=o\left((\log r)^{2-p}\left(\log _{2} r\right)^{-\alpha}\right), \quad 1<p<2, \alpha \in \mathbb{R}, \\
\log M(r, f)-(\log r)\left(\log _{2} r\right)^{\alpha}=o\left((\log r)\left(\log _{2} r\right)^{\min (1-\alpha, 0)}\right), \quad \alpha>0, \\
\log M(r, f)-(\log r)^{2}\left(\log _{2} r\right)^{\alpha}=o\left(\left(\log _{2} r\right)^{|\alpha|}\right), \quad \alpha<0 .
\end{gathered}
$$

(ii) Assertion (i) ceases to be true if one replaces "o" by "O."

The proof of Theorem 1 is based on the following phenomenon, discovered by Hayman in 1966 .

Theorem C (Hayman, [7]). For any transcendental entire function $f$,

$$
\limsup _{r \rightarrow \infty}\left(\frac{d}{d \log r}\right)^{2} \log M(r, f) \geqslant C_{0}
$$

where $C_{0}>0$ is an absolute constant.

In 1974, Boichuk and Goldberg [2] proved that for functions $f$ with nonnegative coefficients the best possible value of $C_{0}$ is $1 / 4$. (For other developments related to Hayman's phenomenon, see Kjellberg [8] and Abi-Khuzam [1].) Ostrovskii found another proof based on a formula due to Rosenbloom [10]. This proof was included in [2]. Now we use the idea of that proof for getting a description of the set where

$$
\left(\frac{d}{d \log r}\right)^{2} \log M(r, f) \geqslant \epsilon^{2}, \quad 0<\epsilon<\frac{1}{2} .
$$

The corresponding Lemma 2.5 below is a crucial point in the proof of Theorem 1 .

\section{Auxiliary Results}

Lemma 2.1. If $w(\log r) \in \mathscr{A}_{\mathrm{reg}}$, then

$$
\lim _{x \rightarrow \infty} w^{\prime \prime}(x)=0 .
$$




\section{Proof. Since}

$$
w^{\prime \prime}(x)=\frac{w(x)}{x^{2}}\left(\lambda^{2}-\lambda+(2 \lambda-1)\left(\vartheta_{1}-\vartheta_{2}\right)^{\prime}(\log x)+\left(\vartheta_{1}-\vartheta_{2}\right)^{\prime 2}(\log x)+\left(\vartheta_{1}-\vartheta_{2}\right)^{\prime \prime}(\log x)\right),
$$

we have, by properties (a)-(c),

$$
w^{\prime \prime}(x)=x^{\lambda-2} e^{\left(\vartheta_{1}-\vartheta_{2}\right)(\log x)}\left(\lambda^{2}-\lambda+(2 \lambda-1)\left(\vartheta_{1}-\vartheta_{2}\right)^{\prime}(\log x)+o\left(\left(\vartheta_{1}+\vartheta_{2}\right)^{\prime}(\log x)\right)\right) .
$$

Therefore, the result is obvious for $1 \leqslant \lambda<2$. For $\lambda=2$, we take into account the fact that $\vartheta_{1} \equiv 0$ and $\vartheta_{2}$ satisfies (a).

Lemma 2.2. One has

$$
\lim _{x \rightarrow \infty} \frac{w^{\prime \prime}(k x)}{w^{\prime \prime}(x)}=k^{\lambda-2},
$$

uniformly on each interval $0<a \leqslant k \leqslant b<\infty$.

Proof. By (8) and (14), we have

$$
\begin{aligned}
\frac{w^{\prime \prime}(k x)}{w^{\prime \prime}(x)}=k^{\lambda-2} & \exp \left(\left(\vartheta_{1}-\vartheta_{2}\right)(\log k x)-\left(\vartheta_{1}-\vartheta_{2}\right)(\log x)\right) \\
& \times \frac{\lambda^{2}-\lambda+(2 \lambda-1)\left(\vartheta_{1}-\vartheta_{2}\right)^{\prime}(\log k x)+o\left(\left(\vartheta_{1}+\vartheta_{2}\right)^{\prime}(\log k x)\right)}{\lambda^{2}-\lambda+(2 \lambda-1)\left(\vartheta_{1}-\vartheta_{2}\right)^{\prime}(\log x)+o\left(\left(\vartheta_{1}+\vartheta_{2}\right)^{\prime}(\log x)\right)} .
\end{aligned}
$$

Using properties (a)-(c) (and, for $\lambda=1$, the fact that $\vartheta_{2} \equiv 0$ ), we obtain the assertion of the lemma.

Lemma 2.3. One has

$$
\lim _{x \rightarrow \infty}\left(x-\frac{w(x)}{w^{\prime}(x)}\right)=\infty .
$$

Proof. It follows from (8) that

$$
x-\frac{w(x)}{w^{\prime}(x)}=\frac{(\lambda-1) x+x\left(\vartheta_{1}-\vartheta_{2}\right)^{\prime}(\log x)}{\lambda+\left(\vartheta_{1}-\vartheta_{2}\right)^{\prime}(\log x)} .
$$

For $\lambda>1$, the assertion of the lemma follows from (b). For $\lambda=1$, we take into account the fact that $\vartheta_{2} \equiv 0$ and note that it suffices to show that $\lim _{x \rightarrow \infty} x \vartheta_{1}^{\prime}(\log x)=\infty$, or, equivalently,

$$
\lim _{t \rightarrow \infty} e^{t} \vartheta_{1}^{\prime}(t)=\infty .
$$

Obviously, (c) implies that $e^{t} \vartheta_{1}^{\prime}(t)$ is nondecreasing. Therefore, if (16) does not hold, then $\vartheta_{1}^{\prime}(t)=$ $O\left(e^{-t}\right), t \rightarrow \infty$. This contradicts (a).

Lemma 2.4. It suffices to prove Theorem 1 under the additional assumption that $f$ has nonnegative Taylor coefficients.

Proof. We derive the lemma from the following theorem.

Theorem D (Erdös-Kövari [5]). Let $f$ be an entire function. There exists an entire function $\hat{f}$ with nonnegative coefficients such that

$$
\frac{1}{6} \leqslant \frac{M(r, f)}{M(r, \hat{f})} \leqslant 3, \quad r \geqslant 0 .
$$

By this theorem, $\log M(r, f)=\log M(r, \hat{f})+O(1)$. Therefore, $f$ satisfies (9) (with "o" or "O") if and only if so does $\hat{f}$.

Let $f$ be an entire transcendental function with nonnegative coefficients,

$$
f(z)=\sum_{k=0}^{\infty} d_{k} z^{k}, \quad d_{k} \geqslant 0, k=0,1,2, \ldots .
$$

Then $M(r, f)=f(r)$. We set

$$
\varphi(x)=f\left(e^{x}\right), \quad \alpha(x)=(\log \varphi(x))^{\prime}, \quad \beta(x)=(\log \varphi(x))^{\prime \prime} .
$$


The function $\alpha(x)$ is nonnegative and increasing and tends to $+\infty$ as $x \rightarrow+\infty$. Therefore, the equations

$$
\alpha\left(a_{k}\right)=k+\varepsilon, \quad \alpha\left(b_{k}\right)=k+1-\varepsilon, \quad k=k_{0}, k_{0}+1, k_{0}+2, \ldots,
$$

where $\varepsilon \in\left(0, \frac{1}{2}\right), k_{0}=[\alpha(0)]+1$, uniquely determine positive numbers

$$
a_{k_{0}}<b_{k_{0}}<a_{k_{0}+1}<b_{k_{0}+1}<\cdots<a_{k}<b_{k}<\rightarrow \infty \quad \text { as } k \rightarrow \infty .
$$

The next lemma plays a crucial role in the proof of Theorem 1 .

Lemma 2.5. Let $f$ be a transcendental entire function with nonnegative coefficients. In the notation introduced above,

$$
\beta(x) \geqslant \varepsilon^{2} \quad \text { for } x \in A_{\varepsilon}:=\bigcup_{k=k_{0}}^{\infty}\left[a_{k}, b_{k}\right]
$$

Proof. We have

$$
\varphi(x)=f\left(e^{x}\right)=\sum_{k=0}^{\infty} d_{k} e^{k x}, \quad d_{k} \geqslant 0, k=0,1,2, \ldots
$$

The following formula is due to Rosenbloom [10]:

$$
\beta(x)=\sum_{k=0}^{\infty}(k-\alpha(x))^{2} \frac{d_{k} e^{k x}}{\varphi(x)} .
$$

Its proof is a direct calculation:

$$
\begin{aligned}
& \alpha(x)=\frac{\varphi^{\prime}(x)}{\varphi(x)}=\sum_{k=0}^{\infty} \frac{k d_{k} e^{k x}}{\varphi(x)} \\
& \beta(x)=\frac{\varphi^{\prime \prime}(x)}{\varphi(x)}-\left(\frac{\varphi^{\prime}(x)}{\varphi(x)}\right)^{2}=\sum_{k=0}^{\infty} \frac{k^{2} d_{k} e^{k x}}{\varphi(x)}-\alpha(x) \sum_{k=0}^{\infty} \frac{k d_{k} e^{k x}}{\varphi(x)}=\sum_{k=0}^{\infty}(k-\alpha(x))^{2} \frac{d_{k} e^{k x}}{\varphi(x)} .
\end{aligned}
$$

Since

$$
\min _{k=0,1,2, \ldots}|k-\alpha(x)| \geqslant \varepsilon
$$

for $x \in A_{\varepsilon}$, the assertion of the lemma follows.

Lemma 2.6. For any $w(\log r) \in \mathscr{A}_{\mathrm{reg}}$, there does not exist an entire function with positive Taylor coefficients such that

$$
\alpha(x)-w^{\prime}(x)=o(1), \quad x \rightarrow \infty .
$$

Proof. Since $w^{\prime \prime}(x) \rightarrow 0$, we have

$$
\beta(x)-w^{\prime \prime}(x)=\left(\alpha(x)-w^{\prime}(x)\right)^{\prime} \geqslant \frac{\varepsilon^{2}}{2}, \quad x \in\left(a_{k}, b_{k}\right), k \geqslant k_{1} .
$$

Using (18) and (20), we obtain

$$
1-2 \varepsilon=\alpha\left(a_{k}\right)-\alpha\left(b_{k}\right)=w^{\prime}\left(a_{k}\right)-w^{\prime}\left(b_{k}\right)+o(1)=w^{\prime \prime}\left(c_{k}\right)\left(b_{k}-a_{k}\right)+o(1),
$$

for some $c_{k} \in\left(a_{k}, b_{k}\right)$. This implies that

$$
b_{k}-a_{k} \rightarrow \infty \text { as } k \rightarrow \infty .
$$

The integration of $(21)$ over $\left(a_{k}, b_{k}\right)$ gives a contradiction to $(20)$. 


\section{Proof of Theorem 1}

In what follows, we agree to denote positive constants by the letter $C$ (with or without subscripts).

Proof of (i). We assume that there exists an entire function $f$ with nonnegative Taylor coefficients which satisfies (9). Let

$$
\log \varphi(x)-w(x)=\tau(x)
$$

where

We claim that

$$
|\tau(x)| \leqslant \epsilon(x) \min \left(1 / w^{\prime \prime}(x), x\right), \quad \epsilon(x) \downarrow 0 .
$$

which contradicts Lemma 2.6.

$$
\alpha(x)-w^{\prime}(x)=o(1), \quad x \rightarrow \infty,
$$

By the convexity of $\log \varphi$, we have the inequality

$$
\alpha(x)(z-x)-[\log \varphi(z)-\log \varphi(x)] \leqslant 0 \text { for any } z \text { and } x .
$$

Therefore, subtracting $w^{\prime}(x)(z-x)-[w(z)-w(x)]$ from both sides, we obtain

$$
\begin{aligned}
{\left[\alpha(x)-w^{\prime}(x)\right](z-x)-[(\log \varphi(z)-w(z))-(\log \varphi(x)-w(x))] } & \leqslant w(z)-w(x)-w^{\prime}(x)(z-x) \\
& =\frac{1}{2} w^{\prime \prime}(c)(z-x)^{2}
\end{aligned}
$$

with some $c$ between $x$ and $z$. We restrict ourselves to $z$ lying in the interval $|z-x| \leqslant x / 2$. Then by Lemma 2.2 there exists a positive constant $C$ such that

$$
\begin{aligned}
{\left[\alpha(x)-w^{\prime}(x)\right](z-x) } & \leqslant 2 \max _{|t-x| \leqslant x / 2}|\tau(t)|+C w^{\prime \prime}(x)(z-x)^{2} \\
& \leqslant C \epsilon(x / 2) \min \left(1 / w^{\prime \prime}(x), x\right)+C w^{\prime \prime}(x)(z-x)^{2} .
\end{aligned}
$$

If $x \leqslant z \leqslant 3 x / 2$, then it follows that

$$
\alpha(x)-w^{\prime}(x) \leqslant \frac{C \epsilon(x / 2) \min \left(1 / w^{\prime \prime}(x), x\right)}{z-x}+C w^{\prime \prime}(x)(z-x) .
$$

We set

$$
z-x=\sqrt{\epsilon(x / 2)} \min \left(1 / w^{\prime \prime}(x), x\right) \leqslant x / 2 .
$$

Then we obtain

$$
\begin{aligned}
\alpha(x)-w^{\prime}(x) & \leqslant C \sqrt{\epsilon(x / 2)}+C w^{\prime \prime}(x) \sqrt{\epsilon(x / 2)} \min \left(1 / w^{\prime \prime}(x), x\right) \\
& =C \sqrt{\epsilon(x / 2)}+C \sqrt{\epsilon(x / 2)} \min \left(1, x w^{\prime \prime}(x)\right) \leqslant C \sqrt{\epsilon(x / 2)}+C \sqrt{\epsilon(x / 2)}=o(1) .
\end{aligned}
$$

If $x / 2 \leqslant z \leqslant x$, then it follows from (22) that

$$
\alpha(x)-w^{\prime}(x) \geqslant \frac{C \epsilon(x / 2) \min \left(1 / w^{\prime \prime}(x), x\right)}{z-x}+C w^{\prime \prime}(x)(z-x) .
$$

By setting

$$
x-z=\sqrt{\epsilon(x / 2)} \min \left(1 / w^{\prime \prime}(x), x\right),
$$

we obtain

$$
\alpha(x)-w^{\prime}(x) \geqslant-(C \sqrt{\epsilon(x / 2)}+C \sqrt{\epsilon(x / 2)})=o(1) .
$$

Proof of (ii). It is straightforward to check that $w^{\prime}(x)$ monotonically increases to $\infty$ for sufficiently large $x$. Therefore, there exists a unique $x_{n}$ such that $w^{\prime}\left(x_{n}\right)=n, n=n_{0}, n_{0}+1, \ldots$. Define positive numbers $c_{n}, n \geqslant n_{0}$, by the equation

$$
\log c_{n}+n x_{n}=w\left(x_{n}\right)
$$

Each of the lines

$$
y_{n}(x):=\log c_{n}+n x, \quad n \geqslant n_{0},
$$


is the tangent to $y=w(x)$ at the respective point $x_{n}$. We set

$$
f(z)=\sum_{k=n_{0}}^{\infty} c_{k} z^{k} .
$$

Since

$$
\frac{\log c_{n}}{n}=-x_{n}+\frac{w\left(x_{n}\right)}{w^{\prime}\left(x_{n}\right)},
$$

it follows from Lemma 2.3 that $\lim _{n \rightarrow \infty}\left|c_{n}\right|^{1 / n}=0$, and hence $f$ is an entire function.

We denote by $\mu(r, f)$ the maximum term of $f$; that is,

$$
\log \mu\left(e^{x}, f\right)=\sup _{n_{0} \leqslant n<\infty}\left(\log c_{n}+n x\right) .
$$

The graph of $\log \mu\left(e^{x}, f\right)$ is a polygonal line lying below the graph of $w(x)$ and touching the latter only for $x=x_{n}$. Thus there exists a $\sigma_{n}, x_{n}<\sigma_{n}<x_{n+1}, n \geqslant n_{0}$, such that

$$
\log \mu\left(e^{x}, f\right)=\log c_{n}+n x, \quad \sigma_{n-1} \leqslant x \leqslant \sigma_{n} .
$$

We shall prove the following:

$$
\begin{gathered}
w(x)-\log \mu\left(e^{x}, f\right)=O\left(\min \left(1 / w^{\prime \prime}(x), x\right)\right), \quad x \rightarrow \infty, \\
\log M\left(e^{x}, f\right)-\log \mu\left(e^{x}, f\right)=O(1), \quad x \rightarrow \infty,
\end{gathered}
$$

from which Theorem 1(ii) readily follows.

First, we prove (23). Note that

$$
\left|\frac{d}{d x}\left(w(x)-\log \mu\left(e^{x}, f\right)\right)\right| \leqslant 1 .
$$

The integration from $x_{n_{0}}$ to $x$ gives

$$
0 \leqslant w(x)-\log \mu\left(e^{x}, f\right) \leqslant x .
$$

By Lemma 2.2, there exists a constant $C_{*}>1$ such that

$$
\frac{1}{C_{*}} \leqslant \frac{w^{\prime \prime}\left(x_{1}\right)}{w^{\prime \prime}\left(x_{2}\right)} \leqslant C_{*} \text { for } \frac{1}{e} \leqslant \frac{x_{1}}{x_{2}} \leqslant e, x_{1}, x_{2} \geqslant x_{0} .
$$

If (23) is violated, then, in view of (25), there exists a $y>e x_{0}$ and a constant $C$ such that

$$
C>\max \left(2 C_{*} e, C_{*}^{3} / 2\right)
$$

and

$$
w(y)-\log \mu\left(e^{y}, f\right) \geqslant \frac{C}{w^{\prime \prime}(y)} .
$$

Using (25), we obtain $w^{\prime \prime}(y) \geqslant C / y$. Then

$$
w^{\prime \prime}(t) \geqslant \frac{w^{\prime \prime}(y)}{C_{*}} \geqslant \frac{C}{C_{*} y} \geqslant \frac{C}{C_{*} e t}
$$

for all $t, y / e \leqslant t \leqslant e y$.

Suppose that $x_{n} \leqslant y<x_{n+1}$. It follows from (28) and (26) that

$$
w^{\prime}(e y)-w^{\prime}(y)=\int_{y}^{e y} w^{\prime \prime}(t) d t \geqslant \frac{C}{C_{*} e}>2 .
$$

This implies $e y \geqslant x_{n+2}$. Likewise, we find that $w^{\prime}(y)-w^{\prime}(y / e)>2$ and therefore $y / e \leqslant x_{n-1}$. Hence we have

If $x_{n} \leqslant y \leqslant \sigma_{n}$, then

$$
\frac{y}{e}<\sigma_{n-1}<x_{n}<\sigma_{n}<x_{n+1}<\sigma_{n+1}<e y .
$$

$$
w(y)-\log \mu\left(e^{y}, f\right)=w(y)-w\left(x_{n}\right)-n\left(y-x_{n}\right)=\frac{1}{2} w^{\prime \prime}(c)\left(y-x_{n}\right)^{2} \leqslant \frac{C_{*}}{2} w^{\prime \prime}(y)\left(y-x_{n}\right)^{2},
$$


where $c \in\left(x_{n}, \sigma_{n}\right)$. In addition,

$$
1 \geqslant w^{\prime}(y)-w^{\prime}\left(x_{n}\right)=\int_{x_{n}}^{y} w^{\prime \prime}(t) d t \geqslant \int_{x_{n}}^{y} \frac{w^{\prime \prime}(y)}{C_{*}} d t=\left(y-x_{n}\right) \frac{w^{\prime \prime}(y)}{C_{*}} .
$$

Combining (29) and (30), we find that

$$
w(y)-\log \mu\left(e^{y}, f\right) \leqslant \frac{C_{*}^{3}}{2 w^{\prime \prime}(y)} .
$$

If $\sigma_{n} \leqslant y<x_{n+1}$, then similar reasoning shows that (31) still holds. But this contradicts (26) and (27).

To show (24), first note that since $w^{\prime \prime}(x) \rightarrow 0$ and $w^{\prime}\left(x_{n+1}\right)-w^{\prime}\left(x_{n}\right)=1$, it follows that $x_{n+1}-x_{n} \rightarrow \infty$ as $n \rightarrow \infty$. Therefore, there exists an $n_{1} \geqslant n_{0}$ such that

$$
x_{n+1}-x_{n} \geqslant 1, \quad n \geqslant n_{1} .
$$

Suppose that $x_{n} \leqslant x<x_{n+1}$. Then

$$
\begin{aligned}
\log \frac{c_{k} e^{k x}}{c_{n} e^{n x}} & =w\left(x_{k}\right)-w\left(x_{n}\right)-k\left(x_{k}-x_{n}\right)+(n-k)\left(x_{n}-x\right) \\
& \leqslant-\int_{x_{k}}^{x_{n}}\left(w^{\prime}(t)-k\right) d t \leqslant-\int_{x_{k+1}}^{x_{n}}\left(w^{\prime}(t)-k\right) d t \leqslant-\int_{x_{k+1}}^{x_{n}} 1 d t \leqslant-(n-k-1)
\end{aligned}
$$

for $n_{1} \leqslant k \leqslant n-1$. Likewise,

$$
\log \frac{c_{k} e^{k x}}{c_{n+1} e^{(n+1) x}} \leqslant \int_{x_{n+1}}^{x_{k}}\left(w^{\prime}(t)-k\right) d t \leqslant \int_{x_{n+1}}^{x_{k-1}}\left(w^{\prime}(t)-k\right) d t \leqslant \int_{x_{n+1}}^{x_{k-1}}(-1) d t \leqslant-(k-n-2)
$$

for $k \geqslant n+2$. Thus

$$
\begin{aligned}
M\left(e^{x}, f\right) & =\sum_{k=n_{0}}^{\infty} c_{k} e^{k x}=\sum_{k=n_{0}}^{n_{1}} c_{k} e^{k x}+\sum_{k=n_{1}+1}^{n-1} c_{k} e^{k x}+c_{n} e^{n x}+c_{n+1} e^{(n+1) x}+\sum_{k=n+2}^{\infty} c_{k} e^{k x} \\
& \leqslant \mu\left(e^{x}, f\right)\left(\sum_{k=n_{0}}^{n_{1}} \frac{c_{k} e^{k x}}{c_{n} e^{n x}}+\sum_{k=n_{1}+1}^{n-1} \frac{c_{k} e^{k x}}{c_{n} e^{n x}}+2+\sum_{k=n+2}^{\infty} \frac{c_{k} e^{k x}}{c_{n+1} e^{(n+1) x}}\right) \\
& \leqslant \mu\left(e^{x}, f\right)\left(O(1)+\sum_{k=n_{1}+1}^{n-1} e^{-(n-k-1)}+\sum_{k=n+2}^{\infty} e^{-(k-n-2)}\right) \leqslant C \mu\left(e^{x}, f\right)
\end{aligned}
$$

for $x_{n} \leqslant x<x_{n+1}$.

\section{References}

[1] F. F. Abi-Khuzam, "Hadamard convexity and multiplicity and location of zeros," Trans. Amer. Math. Soc., 347:8 (1995), 3043-3051.

[2] V. S. Boichuk and A. A. Goldberg, "On the three lines theorem," Mat. Zametki, 15 (1974), $45-53$.

[3] M. L. Cartwright, Integral Functions, Cambridge Tracts in Math. and Math. Physics, vol. 44, Cambridge, at the Univ. Press, Cambridge, 1956.

[4] J. Clunie, T. Kövari, "On integral functions having prescribed asymptotic growth, II," Canad. J. Math., 20 (1968), 7-20.

[5] P. Erdös, T. Kövari, "On the maximum modulus of entire functions," Acta Math. Acad. Sci. Hungar., 7 (1956), 305-317.

[6] A. A. Goldberg, B. Ya. Levin, and I. V. Ostrovskii, "Entire and meromorphic functions," in: Encyclopedia Math. Sci., vol. 85, 1996, 1-193.

[7] W. K. Hayman, "Note on Hadamard's convexity theorem," in: Entire Functions and Related Parts of Analysis (Proc. Sympos. Pure Math., La Jolla, Calif, 1966), Amer. Math. Soc., Providence, R.I., 1968, 210-213. 
[8] B. Kjellberg, "The convexity theorem of Hadamard-Hayman," Proc. Roy. Inst. of Techn., 1973, $87-114$.

[9] B. Ya. Levin, Distribution of Zeros of Entire Functions, Amer. Math. Soc., Providence, R.I., 1980.

[10] P. C. Rosenbloom, "Probability and entire functions," in: Studies in Math. Analysis and Related Topics, Stanford Univ. Press, Stanford, Calif., 1962, 325-332.

[11] G. Valiron, Fonctions entières d'ordre fini et fonctions méromorphes, Monographies de "L'Enseignement Mathématique", vol. 8, Institut de Math., Université, Genève, 1960.

Department of Mathematics, Bilkent University, Ankara, Turkey

Verkin Institute for Low Temperature Physics and Engineering, Kharkov, Ukraine

e-mail: iossif@fen.bilkent.edu.tr

Department of Mathematics, Bilkent University, Ankara, Turkey

e-mail: ureyen@fen.bilkent.edu.tr 